\title{
Control of helical navigation by three-dimensional flagellar beating
}

\author{
Dario Cortese* and Kirsty Y. Wan ${ }^{\dagger}$ \\ Living Systems Institute 86 College of Engineering, Mathematics and Physical Sciences \\ University of Exeter, Exeter EX4 4QD, United Kingdom
}

(Dated: September 27, 2020)

\begin{abstract}
Helical swimming is a ubiquitous strategy for motile cells to generate self-gradients for environmental sensing. The model biflagellate Chlamydomonas reinhardtii rotates at a constant $1-2 \mathrm{~Hz}$ as it swims, but the mechanism is unclear. Here, we show unequivocally that the rolling motion derives from a persistent, non-planar flagellar beat pattern. This is revealed by high-speed imaging and micromanipulation of live cells. We construct a fully-3D model to relate flagellar beating directly to the free-swimming trajectories. For realistic geometries, the model reproduces both the sense and magnitude of the axial rotation of live cells. We show that helical swimming requires further symmetry-breaking between the two flagella. These functional differences underlie all tactic responses, particularly phototaxis. We propose a control strategy by which cells steer towards or away from light by modulating the sign of biflagellar dominance.
\end{abstract}

Organisms perceive their world as three-dimensional. Biological swimmers adopt chiral or helical trajectories to navigate through bulk fluid. Helical navigation is a ubiquitous locomotion strategy found across diverse taxa and multiple length scales, from the planktonic larvae of marine invertebrates [1] to small protists [2] and spermatozoa [3. Bacteria bias the rotation of chiral flagella in response to chemical gradients [4]. In contrast, many unicellular eukaryotes steer towards or away from external stimuli (light, chemicals, gravity) via helical klinotaxis, and a corkscrewing motion around their body axis. Such organisms routinely integrate sensory information obtained by subcellular sensors (receptors, eyespots) which periodically scan the environment, with motor actuators (cilia, flagella), to adjust their swimming trajectories according to the stimulus. These self-actions can enhance signal perception at the microscale [5], with important consequences for evolution and eukaryogenesis [6]. Helical movement could help compensate for asymmetries in body shape [7] and/or filament actuation [8, but it is unclear whether such asymmetries evolved as an adaptation to helical swimming or to facilitate it in the first place. Self-propelled cholesteric liquid crystal droplets also exhibit curling and helical motions, depending on an interplay between surface flows and nematic order 9. The complex motility strategies of real cells can facilitate the development of next-generation artificial swimmers and controllable devices [10].

For algal flagellates, the control of flagellar beating is key to effective spatial navigation and reorientation to photostimuli [11-13]. Spermatozoa steer via changes to their 3D beat pattern [14, while ciliates tilt the orientation of ciliary fields to turn [15]. Chlamydomonas swims with two near-identical flagella using a characteristic breaststroke, rotating slowly $(1-2 \mathrm{~Hz})$ about its axis as it swims along left-handed helices [16, 17]. Each $(\sim 10$ $\mu \mathrm{m})$ cell has a unique photosensor (eyespot), fixed in position on the rigid cell body [18. The eyespot scans the environment and modulates phototactic turning by pe- riodic shading [19, 20. Yet to date, there has been no compelling explanation for the cell's characteristic helical motion. Here, we combine theory and experiment to reveal how Chlamydomonas, despite a very symmetric body plan, manipulates non-planar biflagellar beating to achieve helical swimming and stimulus-dependent steering responses in three dimensions. This is in contrast to other microswimmers which rely on obvious body asymmetries to steer [21], such as two very different types of flagella in the case of dinoflagellates [22].

Model formulation.-We construct a fully-3D model of the swimmer, as three beads interacting hydrodynamically in an incompressible newtonian fluid at zeroReynolds number. The cell body is a sphere of radius $a_{0}$, located at $\boldsymbol{r}_{0}$. Two smaller beads (radius $a \ll a_{0}$ ) are localised to the approximate centers of drag of the flag-

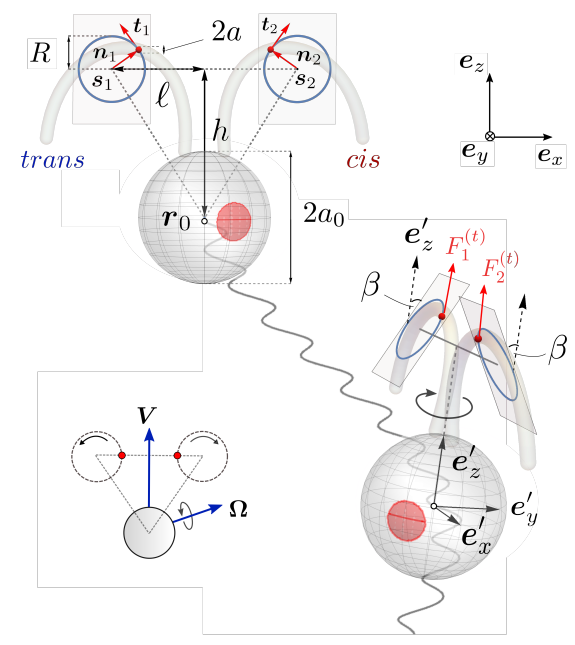

FIG. 1. A fully-3D model of a freely-swimming Chlamydomonas cell, in front, and side views. Flagellar beating is modelled by small beads constrained to rotate along circular orbits embedded within a pair of tilted beat planes, for a realistic scaffold geometry (inset). $\left\{\boldsymbol{e}_{x}^{\prime}, \boldsymbol{e}_{y}^{\prime}, \boldsymbol{e}_{z}^{\prime}\right\}$ and $\left\{\boldsymbol{e}_{x}, \boldsymbol{e}_{y}, \boldsymbol{e}_{z}\right\}$ are the body and laboratory reference frames. 
ella, at $\boldsymbol{r}_{i}, i=1,2$. Flagella beads are driven by variable tangential forces $F_{i}^{(t)}, i=1,2$ and constrained to move along circular orbits of radius $R$ by normal components $F_{i}^{(n)}$, to mimic breaststroke swimming. The 3 beads are constrained on a rigid triangular scaffold assumed to be at rest with the body frame of reference (Fig. 1), with dimensions $\ell$ and $h$, and $a \ll a_{0} \ll h, \ell$. This is motivated by experiments which show that freely-swimming Chlamydomonas cells induce flow-fields that are welldescribed by just three Stokeslets [23. In-plane versions of these models recapitulated the stochastic (runand-tumble) character of biflagellar coordination 24] 27. These minimal representations of ciliary beating as beads moving along a prescribed orbits are powerful tools for studying hydrodynamic synchronization [28 30].

Unlike in previous studies, we allow the flagellar beads to rotate out of the plane. This introduces a non-zero tilt angle $\beta$ between the flagellar orbital planes $\pi_{( \pm \beta)}$ and the frontal $\boldsymbol{e}_{x}^{\prime}-\boldsymbol{e}_{z}^{\prime}$ plane, which we hypothesize suffices to produce both axial rotation and helical swimming (Fig. 1b). The flagella orbits are centered at $\boldsymbol{s}_{i}=\boldsymbol{r}_{0}+(-1)^{i} \ell \boldsymbol{e}_{x}^{\prime}+$ $h \boldsymbol{e}_{z}^{\prime}, i=1,2$. Flagellar beads, located at $\boldsymbol{r}_{i}=\boldsymbol{s}_{i}+R \boldsymbol{n}_{i}$ and $\boldsymbol{n}_{i}=(-1)^{i+1} \cos \varphi_{i} \boldsymbol{e}_{x}^{\prime}+(-1)^{i+1} \sin \beta \sin \varphi_{i} \boldsymbol{e}_{y}^{\prime}+$ $\cos \beta \sin \varphi_{i} \boldsymbol{e}_{z}^{\prime}$, rotate with phases $\varphi_{i}$. For breaststrokes, we require $0 \leq \beta<\pi / 2, \dot{\varphi}_{i} \geq 0$. The body axes $\boldsymbol{e}_{i}^{\prime}$ transform to the lab frame $\boldsymbol{e}_{i}$ via Euler angles $\boldsymbol{\theta}=\left(\theta_{1}, \theta_{2}, \theta_{3}\right)$ (yaw, pitch and roll). The swimmer kinematics are fully described by $\boldsymbol{X}=\left(x_{0}, y_{0}, z_{0}, \theta_{1}, \theta_{2}, \theta_{3}, \varphi_{1}, \varphi_{2}\right)$ and the parameters $h, \ell, \beta, R, a_{0}, a$. We impose force- and torquefree conditions: $\boldsymbol{F}_{0}+\boldsymbol{F}_{1}+\boldsymbol{F}_{2}=\mathbf{0}$ and $\boldsymbol{T}_{0}+\boldsymbol{T}_{1}+\boldsymbol{T}_{2}=\mathbf{0}$, where $\boldsymbol{T}_{i}=\boldsymbol{r}_{i} \times \boldsymbol{F}_{i}+\overline{\boldsymbol{T}}_{i}$, and $\overline{\boldsymbol{T}}_{i}$ is the intrinsic torque due to the $i$ th sphere's rotation around an internal axis. Since $a \ll a_{0}$, we assume that $\overline{\boldsymbol{T}}_{1,2} \ll \overline{\boldsymbol{T}}_{0}$. The swimmer moves with velocity $\boldsymbol{V}=\dot{\boldsymbol{r}}_{0}$, and angular velocity $\boldsymbol{\Omega}$. Using the Oseen approximation for hydrodynamic interactions between beads near a large sphere [31, we have:

$$
\begin{aligned}
\boldsymbol{V} & =\left[\boldsymbol{G}\left(r_{01}\right)-\gamma_{0}^{-1}\right] \cdot \boldsymbol{F}_{1}+\left(\boldsymbol{G}\left(r_{02}\right)-\gamma_{0}^{-1}\right) \cdot \boldsymbol{F}_{2} \\
\dot{\boldsymbol{r}}_{1} & =\gamma_{1}^{-1} \boldsymbol{F}_{1}+\left[\boldsymbol{G}\left(r_{12}\right)-\boldsymbol{G}\left(r_{10}\right)\right] \cdot \boldsymbol{F}_{2}-\boldsymbol{G}\left(r_{10}\right) \cdot \boldsymbol{F}_{1} \\
\dot{\boldsymbol{r}}_{2} & =\gamma_{2}^{-1} \boldsymbol{F}_{2}+\left[\boldsymbol{G}\left(r_{21}\right)-\boldsymbol{G}\left(r_{20}\right)\right] \cdot \boldsymbol{F}_{1}+\boldsymbol{G}\left(r_{20}\right) \cdot \boldsymbol{F}_{2} \\
\boldsymbol{\Omega} & =\gamma_{0 r}^{-1} \overline{\boldsymbol{T}}_{0}=-\gamma_{0 r}^{-1}\left(\boldsymbol{T}_{1}+\boldsymbol{T}_{2}\right) .
\end{aligned}
$$

where $\boldsymbol{r}_{i j}=\boldsymbol{r}_{i}-\boldsymbol{r}_{j}, \gamma_{i}=6 \pi \eta a_{i}, \gamma_{0 r}=8 \pi \eta a_{0}, \boldsymbol{G}(\boldsymbol{r})=$ $(\mathbb{I}+\hat{\boldsymbol{r}} \otimes \hat{\boldsymbol{r}}) /(8 \pi \eta)$, and dot denotes a time derivative. These reduce to a set of 10 equations for unknowns $\left(\boldsymbol{V}, \boldsymbol{\Omega}, \dot{\varphi}_{1,2}, F_{1,2}^{(n)}\right)$ 31]. The method of quaternions was used to resolve singularities at $\theta_{2}= \pm \pi / 2$ [32].

Experiments.- Our model aims to link the threedimensional nature of flagellar beating to the cell's freeswimming behaviour, but is Chlamydomonas flagellar beating truly non-planar, and if so, to what extent? A possible out-of-plane component was detected based on manual tracings of flagellar waveforms [16, but remains unclear. The same authors argued that helical swimming could result from transient asynchronies between the two flagella. Yet this is incompatible with more recent studies showing that asynchronies, or phase-slips, occur randomly, not periodically [26, 35. We will use live-cell imaging and micromanipulation to prove that Chlamydomonas flagella beat with a persistent, non-planar pattern in vivo. To better visualise flagella motion, we aspirate cells onto pipettes to prevent body motion (following previous protocols [35, 36]). Cells are repositioned so that flagella are viewed (at $3000 \mathrm{fps}$ ) directly from above, with the eyespot delineating the cis (the flagellum closest to the eyespot) and trans flagella (Fig. 2a).

Our main findings are threefold. First, viewed from above the two basal bodies are displaced by a small clockwise offset, as reported previously in fixed specimens imaged using electron microscopy [37. This offset is most evident when flagella motion is tracked by optical flow and overlaid to visualise the extent of movement (Fig. 2c). Second, the waveform is inherently non-planar. The Chlamydomonas power-recovery stroke cycle is usually imaged in the $\boldsymbol{e}_{x}^{\prime}-\boldsymbol{e}_{z}^{\prime}$ plane (Fig. 2c-inset), here we re-
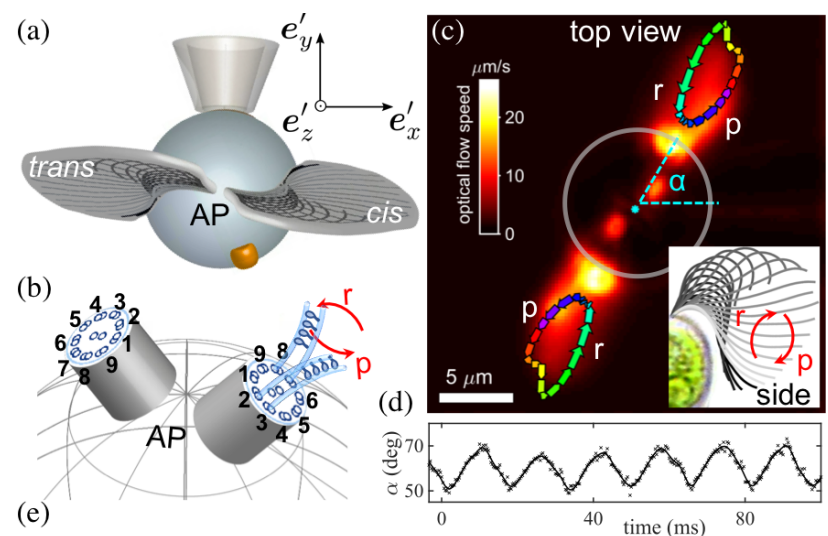

(e)

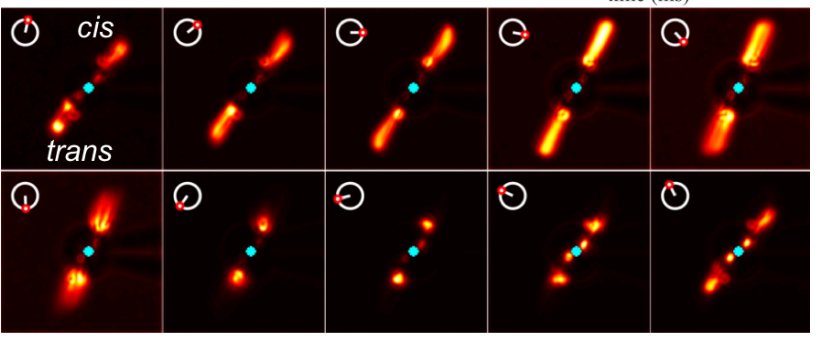

FIG. 2. The non-planar beat pattern of Chlamydomonas flagella (see SM Video 1). (a) Cells are aspirated onto pipettes and imaged from the anterior pole (AP). An eyespot is located $\sim 45^{\circ}$ from the mean beat plane. (b) Basal bodies have a pre-defined symmetry with respect to axonemal microtubule doublets, numbered $1 \rightarrow 9$. Dyneins are separated into two groups, those on doublets operate 2,3,4 for the power (p) stroke, but $6,7,8$ for the recovery (r) stroke 33. (c) Waveforms are tracked by optic flow (arrows show direction of tip rotation), showing the relative offset between the basal bodies and the tilted beat planes. The beat plane (defined by $\alpha$ ) rotates periodically $(\mathrm{d})$. Waveforms are ordered by phase [34] and averaged over $\sim 1000$ consecutive cycles to show the phase-dependence of the non-planar beat pattern (e). 

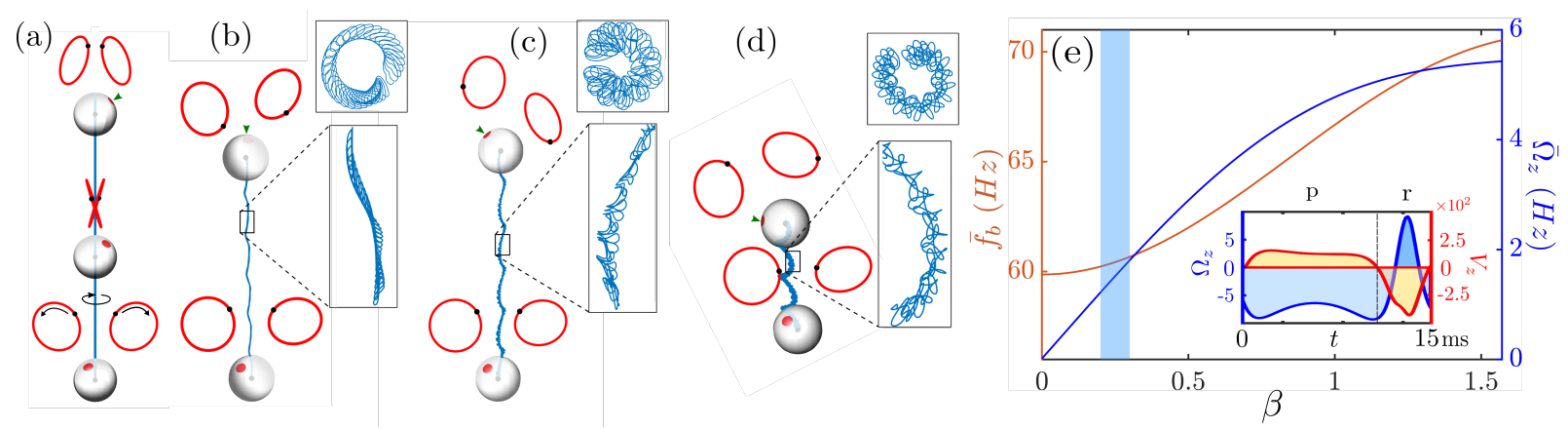

FIG. 3. Non-planar flagellar beating leads to superhelical trajectories (eyespot: small arrowhead). For the same geometry $a_{0}=0.53, h=1.30, R=0.60, \beta=0.30, a=0.05$, examples show (a) purely axial-rotation when $c_{1,2}=0.7$, (b) 'smooth' superhelix for $\left.c_{1}=0.7, c_{2}=0.71, \mathrm{c}\right)$ a superhelix with irregular inner helix for asymmetrical driving forces $c_{1}=0.6, c_{2}=0.62$, (d) a more irregular trajectory for $c_{1}=0.3, c_{2}=0.5$. (e) Mean beat frequency of the two flagella (orange) and axial component of angular velocity (blue) as functions of $\beta$ (numerical data fitted with sum of sines). The shaded region marks range of $\beta$ measured in experiments [31. Inset: linear and angular velocities (in $\mu \mathrm{m} / \mathrm{s}$ and $\mathrm{Hz}$ ) over one beat, note the CW-forward motion during the power (p) stoke and CCW-backward motion during the recovery (r) stroke.

veal that there is also a significant component of motion in the $\boldsymbol{e}_{x}^{\prime}-\boldsymbol{e}_{y}^{\prime}$ plane (Figure 2c-e, SM Video 1). During the power stroke, the flagella extend and pull away from the body while flagellar tips move in opposite directions; the direction of travel is immediately reversed during each subsequent recovery stroke. Over a stereotypical beat, the tips trace closed orbits (Fig. 2c). The rotary motion generates axial torques, balanced here by the micropipette. A freely-swimming cell, viewed from above, will therefore rotate clockwise $(\mathrm{CW})$ during the power stroke but counter-clockwise (CCW) during the recovery stroke. This is analogous to the "two steps forward, one step backward" interpretation for the in-plane breaststroke, which arises due to flagella drag anisotropy. Third, the rotary motion is periodic (coincident with beat frequency), and is stable over thousands of cycles. To estimate the effective orbital tilt angle $\beta$ from the highspeed videos, we use the following trignometric expression $\beta=\sin ^{-1}\left(\frac{\ell}{R} \tan \frac{\Delta \alpha}{2}\right)$, where $\Delta \alpha$ is the angle subtended by the flagellum's uppermost tip in the transverse $\boldsymbol{e}_{x}^{\prime}-\boldsymbol{e}_{y}^{\prime}$ plane [31]. For $N=6$ distinct cells, $\sim 10^{3}$ consecutive beat cycles per individual, $\beta=0.3 \mathrm{rad} \approx 17.2^{\circ}$ (see summary statistics in Table 1 [31]).

Simulations. - We input this estimate of $\beta$ into our 3-bead model with cell-realistic parameters to determine if beat non-planarity can indeed generate axial rotation and helical swimming. All lengths are nondimensionalised by $\ell$ (=10 $\mu \mathrm{m}$, typical cell size), forces by the average tangential flagellar force $F_{0}(=30 \mathrm{pN}$, typical force produced by a flagellum [38], and $\eta$ by $10^{-3} \mathrm{pN} \mu \mathrm{m}^{-2}$ (viscosity of water). When $\beta=0$ (no tilt), pitch and roll $\left(\theta_{2,3}\right)$ are suppressed, and our model reduces to the purely planar case investigated by other authors 24, 39. This is when the only non-vanishing component of torque is along $\boldsymbol{e}_{y}^{\prime}$ (equation (4)) and swimming is constrained to the cell's frontal plane $\left(\Omega_{y}\right.$, yaw).
For a non-planar beat $(\beta \neq 0)$, there is a non-vanishing resultant axial torque $T_{z}$. When flagellar driving forces are equal $\left(F_{1}^{(t)}=F_{2}^{(t)}\right)$, we have purely axial rotation, since other components of $\boldsymbol{\Omega}$ cancel by symmetry. The resulting trajectory is linear, with $\boldsymbol{\Omega} \| \boldsymbol{V}$. The trajectory can be a non-degenerate helix only if $\boldsymbol{\Omega} \nVdash \boldsymbol{V}$. Rotational symmetry about $\boldsymbol{e}_{z}^{\prime}$ can be broken if (1) the scaffold shape is asymmetric; (2) flagella planes have different tilt $\left(\beta_{1} \neq \beta_{2}\right)$; (3) flagella experience different driving forces $\left(F_{1}^{(t)} \neq F_{2}^{(t)}\right)$. The first two cases sometimes produced trajectories that were irregular and non-helical [31. Since we could not detect any obvious morphological asymmetries between the two Chlamydomonas flagella, we focus on case 2 , and only consider asymmetric force profiles of the form $F_{i}^{(t)}=1+c_{i} \cos \varphi_{i}$ [30, 40].

For $\beta>0$, symmetry-breaking introduced by hydrodynamic interactions between the beads results in either forward-CW or backward-CCW swimming at different phases of the beat cycle. The cycle-averaged swimming speed is given by $\overline{\boldsymbol{V}}=\frac{1}{T_{b}} \int_{0}^{T_{b}} \boldsymbol{V}(t) d t=\frac{1}{T_{b}} \int_{0}^{2 \pi} \frac{\boldsymbol{V}(\bar{\varphi})}{\bar{\varphi}} d \bar{\varphi}$ with flagellar period $T_{b}$ and $\bar{\varphi}=\left(\varphi_{1}+\varphi_{2}\right) / 2$, with an analogous expression for angular velocity $\bar{\Omega}$ [31. The dynamical system described by equations (2)-(3) is highly sensitive to swimmer shape. The strength of the hydrodynamic interactions between flagella beads are dictated by scaffold parameters, which determine net swimming (and rotation) direction per cycle, as in the 2D case [25, 40]. We choose configurations where $\bar{V}_{z}>0, \bar{\Omega}_{z}<0$ (CCW is positive), and average flagella beat frequencies $f_{b}$ and axial rotation $\bar{\Omega}_{z}$ fall within experimental values.

We simulate free-swimming trajectories (Fig. 3) for a fixed scaffold shape $\left(a_{0}=0.53, h=1.30, R=0.60\right.$, $a=0.05$ ), tilt angle $\beta=0.3$, but different values of force asymmetry $\Delta F=c_{2}-c_{1}$. Fig. 3a is for purely axial rotation $(\Delta F=0)$. When $\Delta F \neq 0$, trajectories are $s u$ perhelical. Superhelices emerge as general solutions of 
all Low-Re swimming dynamics driven by periodic deformations [11, and have been observed in sperm swimming and in biaxial self-propelled particles under external torques [41]. A $1 \%$ difference in $\Delta F$ suffices to generate realistic superhelical trajectories (Fig. 3b). Here, the average trajectory of the centroid $\mathbf{r}_{\mathbf{0}}$ prescribes an outer helix with a pitch of $\sim 95 \mu \mathrm{m}$ and radius $\sim 5.9 \mu \mathrm{m}$, while "fast" helical swirls appear on the time scale of the flagellar period $T_{b}=f_{b}^{-1} \approx 17 \mathrm{~ms}$. Less regular helices emerge with higher asymmetry (Fig. 3c,d). Frequencies of mean flagellar bead rotation $f_{b}$ and axial rotation $\Omega_{z}$ vary with increasing orbital tilt angle $\beta \in(0,2 \pi)$ (Fig. $3 \mathrm{e})$. As expected, axial rotation velocity increases with beat non-planarity (higher $\beta$ ). A tilt angle $\beta \approx 0.3$ corresponds to a rotation frequency of 1-2 Hz. We conclude that realistic helical swimming with a symmetric scaffold shape can be achieved using a very small force asymmetry between flagella, together with a small orbital tilt.

Phototactic steering. - Can cells exploit asymmetric flagellar driving for trajectory control? We hypothesize that this mechanism underlies phototaxis - directed movement toward/away from light. Chlamydomonas cells perform positive or negative phototaxis, depending on the nature of the stimulus [19, 42. Phototactic steering is associated with changes in both beat amplitude and frequency and is fine-tuned to the body rotation frequency [16, 20, 43]. In general, steering is accomplished by changing $\Omega$ [10, 44, which in turn changes helix properties (radius, pitch, orientation). Here, $\boldsymbol{\Omega}$ and $f_{b}$ are strongly coupled. To simulate phototaxis (Fig. 4), we assume that a light stimulus I either attenuates or accentuates $\Delta F$ depending on the alignment between the eyespot and stimulus [45]. Denoting by $\hat{\mathbf{N}}$ the vector normal to the eyespot surface, the intensity incident on the eyespot is $I(t)=I_{0}(t) \cos \phi(t) H(\cos \phi(t))$, where $\cos \phi=\hat{\boldsymbol{N}} \cdot \hat{\boldsymbol{I}}=\left(\boldsymbol{e}_{x}^{\prime}-\boldsymbol{e}_{y}^{\prime}\right) \cdot\left(-\boldsymbol{e}_{y}\right) / \sqrt{2}$, and $H$ is the Heaviside step function. This framework can be applied to any type of taxis in response to a vectorial cue.

Flagella identity is important. When $\Delta F=0.01$ (no signal), the stronger (dominant) flagellum is the cis flagellum $\left(c_{\text {cis }}^{(0)}=0.71, c_{\text {trans }}^{(0)}=0.7\right)$. Force profiles are then modified to $c_{\text {cis, trans }}=c_{\text {cis,trans }} \mp p \log (1+I(t))$, where $p$ is the sensitivity of biflagellar dominance to the signal. Signs were chosen so that the model flagella responded differentially to the same signal [18, and in a direction compatible with experiments [46. Importantly, here $\boldsymbol{\Omega}$ emerges from the biflagellar force profiles, and was not assumed a priori to produce a regular helix. The resulting dynamics are characterised by a change in the pitch, radius and axis of the (super)helical trajectory. The sign of flagellar dominance, determines the sign of phototaxis. Compared to the no-light scenario, the cell turns towards the light when $\Delta F$ flips sign (trans-dominant), but away when the cis-flagellum becomes more dominant ( $\Delta F$ more positive). This indicates that alignment to a
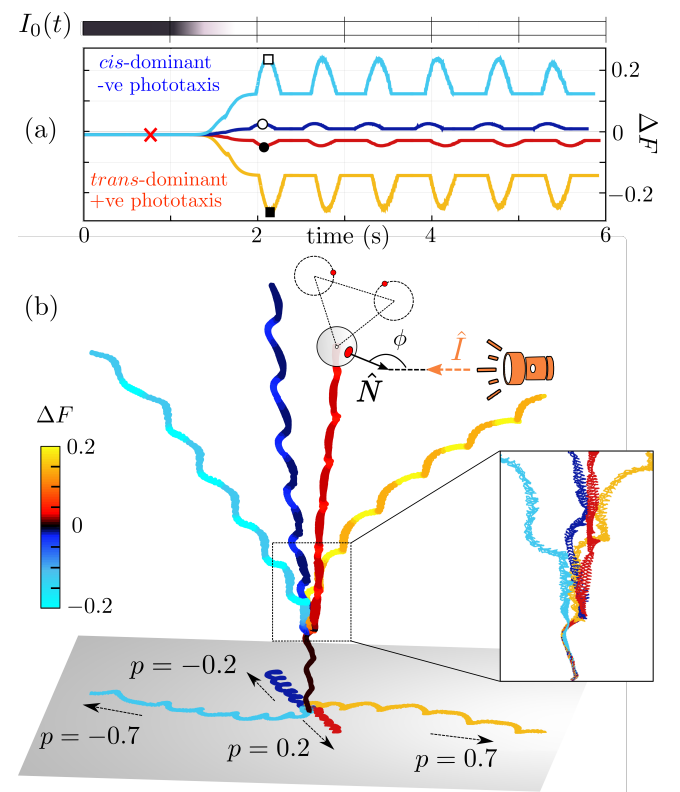

FIG. 4. Flagellar dominance controls phototactic steering. (a) Time-series of stimulus intensity $I_{0}$ (grayscale $=$ intensity) and the response $\Delta F$ for four different $p$ values. Markers indicate characteristic trans-cis frequencies: $\times: 61-63 \mathrm{~Hz}$, $\square: 58-75 \mathrm{~Hz}, \mathbf{\square}: 78-51 \mathrm{~Hz}, \circ: 51-73 \mathrm{~Hz}, \bullet: 53-69 \mathrm{~Hz}$. (b) The corresponding $3 \mathrm{D}$ tracks show either positive or negative phototaxis. Color indicates force asymmetry $\Delta F$. In all cases, $a_{0}=0.53, h=1.3, \ell=1, R=0.6, \beta=0.3, a=0.05, c_{\text {cis }}(t=$ $0)=0.71, c_{\text {trans }}(0)=0.7$.

vectorial stimulus is indeed possible simply by varying the two relative forcing profiles (Fig. 4).

Concluding remarks. - We showed for the first time that Chlamydomonas flagella beat with a persistent, 3D beat pattern in vivo, and quantified the extent of this non-planarity in terms of an orbital tilt. This complements a recent study which revealed that isolated axonemes, when reactivated with ATP, beat with a threedimensional pattern [47. It would seem that perfectly planar beats cannot be generated by an intrinsically chiral axoneme (Fig. 2b) [11, 48. In Chlamydomonas, while the out-of-plane tilt may be fixed genetically, the in-plane beat pattern is dynamic 35]. Accounting for this 3D beat, we developed a three-bead hydrodynamic model with cell-realistic geometries which captures the sense and magnitude of axial rotation of real cells. Superhelical trajectories emerged directly from the flagellar motion, and were not prescribed. The model swims more slowly $(15-30 \mu \mathrm{m} / \mathrm{s})$ than live cells $(50-100 \mu \mathrm{m} / \mathrm{s}$ [4] $)$, suggesting limitations of a Stokeslet-type swimmer. Here, beat patterns/frequencies can only be changed by variable forcing, so that incorporating the full slender-filament dynamics will better mimic the amplitude-frequency coupling pertaining to real flagella. Basal coupling 50] may be additionally required to constrain biflagellar synchrony to enhance swimming and steering efficiency. 
A small asymmetry (1\%) in biflagellar driving forces was necessary and sufficient for helical (not just purely axial) swimming. Whether this is augmented by further structural asymmetries (if $\beta_{1} \neq \beta_{2}$ ) can be revealed by multi-focal imaging, to measure the full torsional profile of each flagellum. Our simple three-bead swimmer was able to perform phototaxis, requiring only a stimulusdependent source of asymmetry. Controllable steering is thus fairly insensitive to gait and the precise beating waveform. Indeed some algal biflagellates which do not use in-phase breaststrokes are nonetheless phototactic (e.g. Polytoma, Chryptomonas). Our work emphasizes the functional distinction between the two Chlamydomonas flagella, showing that a tunable and reversible biflagellar dominance likely operates in live cells for helical klinotaxis. Consistent with this view, mutant strains (mia, bop5-3, ptx1, and lsp1) have 'smoother' trajectories, and defective or weakened phototaxis [51 53. Either flagellum can be dominant, depending on the stimulus. Indeed $\mathrm{Ca}^{2+}$ has been shown to produce opposite responses in reactivated cis and trans axonemes [18, 46]. Our results offer key insights into the physiological basis of dynamic sensorimotor control, as implemented in a simple, aneural organism with few morphological asymmetries. An important next step will be to determine how biflagellar dominance is regulated at the molecular level (e.g. of dynein activity).

We thank Ray Goldstein for financial support during the initial phase of this project, and Peter Ashwin and Gáspár Jékely for useful discussions. KYW is grateful for funding from an Academy of Medical Sciences Springboard Award, and the European Research Council (ERC) under the European Union's Horizon 2020 research and innovation programme (grant no. 853560, EvoMotion).

* d.cortese@exeter.ac.uk

$\dagger$ k.y.wan2@exeter.ac.uk

[1] G. Jékely, J. Colombelli, H. Hausen, K. Guy, E. Stelzer, F. Nédélec, and D. Arendt, Mechanism of phototaxis in marine zooplankton, Nature 456, 395 (2008).

[2] T. L. Jahn and J. J. Votta, Locomotion of Protozoa, Annu. Rev. Fluid Mech. 4, 93 (1972).

[3] A. D. Drake, Observations on bull sperm rotation, Biology of Reproduction 10, 78 (1974)

[4] E. Lauga, Bacterial Hydrodynamics, Annual Review of Fluid Mechanics 48, 105 (2016).

[5] B. M. Friedrich and F. Jülicher, Steering chiral swimmers along noisy helical paths, Phys. Rev. Lett. 103, 068102 (2009)

[6] K. Y. Wan and G. Jékely, Origins of eukaryotic excitability, https://arxiv.org/abs/2007.13388 (2020).

[7] H. S. Jennings, On the significance of the spiral swimming of organisms, American Naturalist 35, 369 (1901)

[8] H. Gadêlha, P. Hernández-Herrera, F. Montoya,
A. Darszon, and G. Corkidi, Human sperm uses asymmetric and anisotropic flagellar controls to regulate swimming symmetry and cell steering, Science Advances 6, 10.1126/sciadv.aba5168 (2020).

[9] C. Krüger, G. Klös, C. Bahr, and C. C. Maass, Curling Liquid Crystal Microswimmers: A Cascade of Spontaneous Symmetry Breaking, Phys. Rev. Lett. 117, 048003 (2016).

[10] F. Lancia, T. Yamamoto, A. Ryabchun, T. Yamaguchi, M. Sano, and N. Katsonis, Reorientation behavior in the helical motility of light-responsive spiral droplets, Nature Communications 10, 5238 (2019).

[11] M. Rossi, G. Cicconofri, A. Beran, G. Noselli, and A. De Simone, Kinematics of flagellar swimming in Euglena gracilis: Helical trajectories and flagellar shapes, PNAS 114, 13085 (2017)

[12] A. C. H. Tsang, A. T. Lam, and I. H. Riedel-Kruse, Polygonal motion and adaptable phototaxis via flagellar beat switching in the microswimmer Euglena gracilis, Nature Physics 14, 1216 (2018)

[13] H. De Maleprade, F. Moisy, T. Ishikawa, and R. E. Goldstein, Motility and phototaxis of gonium, the simplest differentiated colonial alga, Physical Review E 101, 022416 (2020).

[14] A. Gong, S. Rode, U. B. Kauppl, G. Gompper, J. Elgeti, B. M. Friedrich, and L. Alvarez, The steering gaits of sperm, Philosophical Transactions of the Royal Society B-biological Sciences 375, 20190149 (2020).

[15] H. Ito, T. Omori, and T. Ishikawa, Swimming mediated by ciliary beating: comparison with a squirmer model, Journal of Fluid Mechanics 874, 774-796 (2019)

[16] U. Rüffer and W. Nultsch, High-speed cinematographic analysis of the movement of Chlamydomonas, Cell Motility 5, 251 (1985)

[17] K. Schaller, R. David, and R. Uhl, How Chlamydomonas keeps track of the light once it has reached the right phototactic orientation, Biophys. J. 73, 1562 (1997)

[18] R. Kamiya and G. B. Witman, Submicromolar levels of calcium control the balance of beating between the two flagella in demembranated models of Chlamydomonas., The Journal of Cell Biology 98, 97 (1984).

[19] K. W. Foster and R. D. Smyth, Light Antennas in phototactic algae., Microbiol Rev 44, 572 (1980).

[20] K. Josef, J. Saranak, and K. W. Foster, Ciliary behavior of a negatively phototactic chlamydomonas reinhardtii, Cell Motility and the Cytoskeleton 61, 97 (2005).

[21] R. J. Wheeler, Use of chiral cell shape to ensure highly directional swimming in trypanosomes, Plos Computational Biology 13, e1005353 (2017).

[22] T. O. M. Fenchel, How Dinoflagellates Swim, Protist 152, 329 (2001)

[23] K. Drescher, R. E. Goldstein, N. Michel, M. Polin, and I. Tuval, Direct Measurement of the Flow Field around Swimming Microorganisms, Phys. Rev. Lett. 105, 168101 (2010)

[24] R. R. Bennett and R. Golestanian, Emergent Run-andTumble Behavior in a Simple Model of Chlamydomonas with Intrinsic Noise, Phys. Rev. Lett. 110, 148102 (2013).

[25] K. Polotzek and B. M. Friedrich, A three-sphere swimmer for flagellar synchronization, New Journal of Physics 15, 045005 (2013).

[26] R. E. Goldstein, M. Polin, and I. Tuval, Noise and synchronization in pairs of beating eukaryotic flagella, Physical review letters 103, 168103 (2009). 
[27] E. Lushi, V. Kantsler, and R. E. Goldstein, Scattering of biflagellate microswimmers from surfaces, Physical Review E 96, 023102 (2017).

[28] A. Vilfan and F. Jülicher, Hydrodynamic Flow Patterns and Synchronization of Beating Cilia, Phys. Rev. Lett. 96, 058102 (2006).

[29] N. Bruot and P. Cicuta, Realizing the Physics of Motile Cilia Synchronization with Driven Colloids, Annual Review of Condensed Matter Physics 7, 323 (2016).

[30] D. R. Brumley, M. Polin, T. J. Pedley, and R. E. Goldstein, Hydrodynamic synchronization and metachronal waves on the surface of the colonial alga volvox carteri, Physical review letters 109, 268102 (2012).

[31] See supplemental material at [url will be inserted by the publisher] for further details and results., .

[32] M. Boyle, The Integration of Angular Velocity, Adv. Appl. Clifford Algebras 27, 2345 (2017).

[33] H. J. Hoops and G. B. Witman, Outer doublet heterogeneity reveals structural polarity related to beat direction in chlamydomonas flagella., The Journal of cell biology 97, 902 (1983).

[34] K. Y. Wan and R. E. Goldstein, Rhythmicity, recurrence, and recovery of flagellar beating, Physical Review Letters 113, 238103 (2014).

[35] K. Y. Wan, K. C. Leptos, and R. E. Goldstein, Lag, lock, sync, slip: the many 'phases' of coupled flagella, Journal of the Royal Society Interface 11, 20131160 (2014)

[36] K. Y. Wan and R. E. Goldstein, Coordinated beating of algal flagella is mediated by basal coupling, Proceedings of the National Academy of Sciences 113, E2784 (2016).

[37] N. Yubuki and B. S. Leander, Evolution of microtubule organizing centers across the tree of eukaryotes, The Plant Journal 75, 230 (2013).

[38] T. J. Böddeker, S. Karpitschka, C. T. Kreis, Q. Magdelaine, and O. Bäumchen, Dynamic force measurements on swimming chlamydomonas cells using micropipette force sensors, Journal of the Royal Society Interface 17, 20190580 (2020).

[39] B. M. Friedrich and F. Jülicher, Flagellar Synchronization Independent of Hydrodynamic Interactions, Phys. Rev. Lett. 109, 138102 (2012)

[40] R. R. Bennett and R. Golestanian, Phase-dependent forcing and synchronization in the three-sphere model of Chlamydomonas, New J. Phys. 15, 075028 (2013)

[41] R. Wittkowski and H. Löwen, Self-propelled Brownian spinning top: Dynamics of a biaxial swimmer at low Reynolds numbers, Phys. Rev. E 85, 021406 (2012).

[42] K.-i. Wakabayashi, Y. Misawa, S. Mochiji, and R. Kamiya, Reduction-oxidation poise regulates the sign of phototaxis in chlamydomonas reinhardtii, Proceedings of the National Academy of Sciences 108, 11280 (2011).

[43] K. C. Leptos, M. Chioccioli, S. Furlan, A. I. Pesci, and R. E. Goldstein, An Adaptive Flagellar Photoresponse Determines the Dynamics of Accurate Phototactic Steering in Chlamydomonas, bioRxiv , 254714 (2018)

[44] H. C. Crenshaw, Orientation by helical motion-I. Kinematics of the helical motion of organisms with up to six degrees of freedom, Bulletin of Mathematical Biology 55, 197 (1993)

[45] R. R. Bennett and R. Golestanian, A steering mechanism for phototaxis in Chlamydomonas, Journal of The Royal Society Interface 12, 20141164 (2015)

[46] N. Isogai, R. Kamiya, and K. Yoshimura, Dominance between the two flagella during phototactic turning in chlamydomonas, Zoological Science 17, 1261 (2000).

[47] S. Mojiri, S. Isbaner, S. Mühle, H. Jang, A. J. Bae, I. Gregor, A. Gholami, and J. Enderlein, Three-dimensional beating dynamics of chlamydomonas flagella, bioRxiv 10.1101/2020.07.20.212159 (2020).

[48] P. Sartori, V. F. Geyer, A. Scholich, F. Jülicher, and J. Howard, Dynamic curvature regulation accounts for the symmetric and asymmetric beats of Chlamydomonas flagella, eLife 5, 10.7554/eLife.13258 (2016).

[49] V. A. Martinez, R. Besseling, O. A. Croze, J. Tailleur, M. Reufer, J. Schwarz-Linek, L. G. Wilson, M. A. Bees, and W. C. Poon, Differential dynamic microscopy: A high-throughput method for characterizing the motility of microorganisms, Biophysical journal 103, 1637 (2012).

[50] K. Y. Wan and R. E. Goldstein, Coordinated beating of algal flagella is mediated by basal coupling, PNAS 113, E2784 (2016)

[51] K. E. VanderWaal, R. Yamamoto, K.-i. Wakabayashi, L. Fox, R. Kamiya, S. K. Dutcher, P. V. Bayly, W. S. Sale, and M. E. Porter, bop5 mutations reveal new roles for the ic138 phosphoprotein in the regulation of flagellar motility and asymmetric waveforms, Molecular biology of the cell 22, 2862 (2011).

[52] K. C. Leptos, K. Y. Wan, M. Polin, I. Tuval, A. I. Pesci, and R. E. Goldstein, Antiphase Synchronization in a Flagellar-Dominance Mutant of Chlamydomonas, Phys. Rev. Lett. 111, 158101 (2013)

[53] N. Okita, N. Isogai, M. Hirono, R. Kamiya, and K. Yoshimura, Phototactic activity in Chlamydomonas 'non-phototactic' mutants deficient in $\mathrm{Ca} 2+$-dependent control of flagellar dominance or in inner-arm dynein, Journal of Cell Science 118, 529 (2005). 